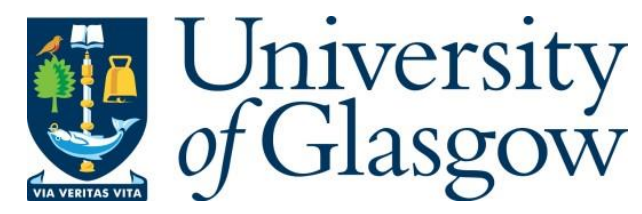

Meder, M., Rapp, A., Plumbaum, T. and Hopfgartner, F. (2017) DataDriven Gamification Design. In: Academic MindTrek, Tampere, Finlanc, 20-21 Sept 2017, pp. 255-258. ISBN 9781450354264

(doi:10.1145/3131085.3131116)

This is the author's final accepted version.

There may be differences between this version and the published version. You are advised to consult the publisher's version if you wish to cite from it.

http://eprints.gla.ac.uk/146799/

Deposited on: 29 August 2017

Enlighten - Research publications by members of the University of Glasgow http://eprints.gla.ac.uk 


\title{
Data-Driven Gamification Design
}

\author{
Short Paper
}

\author{
Michael Meder \\ Technische Universität Berlin \\ Berlin, Germany \\ meder@dai-labor.de \\ Till Plumbaum \\ Technische Universität Berlin \\ Berlin, Germany \\ till@dai-labor.de
}

\author{
Amon Rapp \\ University of Torino \\ Torino, Italy \\ amon.rapp@gmail.com \\ Frank Hopfgartner \\ University of Glasgow \\ Glasgow, United Kingdom \\ frank.hopfgartner@glasgow.ac.uk
}

\begin{abstract}
Gamification has been attracted much interest, not only in the $\mathrm{HCI}$ community, in the last few years. However, there is still a lack of insights and theory on the relationships between game design elements, motivation, domain context and user behavior. In this workshop we want to discover the potentials of data-driven gamification design optimization, e.g. by the application of machine learning techniques on user interaction data in a certain domain.
\end{abstract}

\section{KEYWORDS}

Gamification, User Interaction Data, Artificial Intelligence, Machine Learning

\section{ACM Reference format:}

Michael Meder, Amon Rapp, Till Plumbaum, and Frank Hopfgartner. 2017. Data-Driven Gamification Design. In Proceedings of AcademicMindtrek'17, Tampere, Finland, September 20-21, 2017, 4 pages.

DOI: $10.1145 / 3131085.3131116$

\section{MOTIVATION}

Over the last years, non-recreational services and applications that make use of game elements grew in popularity. Gamification established as a design techniques to increase the user engagement, and change behavior in a variety of contexts $([1,2,16])$. However, study after study researchers keep to ask: "Does Gamification Work?" [6]. Currently the answer should be: it depends. The current state of the art in gamification design consists of several black boxes affecting each other: a set of game design elements, different user types, and diverse contexts. Several already existing gamification design frameworks [4] provide recommendations on how to map different user types to different game design elements or list different categories of game design elements which should be considered for an application of gamification. However, it seems that we still lack of a thorough and clear knowledge about what kinds of game elements

Permission to make digital or hard copies of all or part of this work for personal or classroom use is granted without fee provided that copies are not made or distributed for profit or commercial advantage and that copies bear this notice and the full citation on the first page. Copyrights for components of this work owned by others than ACM must be honored. Abstracting with credit is permitted. To copy otherwise, or republish, to post on servers or to redistribute to lists, requires prior specific permission and/or a fee. Request permissions from permissions@acm.org.

AcademicMindtrek'17, Tampere, Finland

(C) 2017 ACM. 978-1-4503-5426-4/17/09...\$15.00

DOI: $10.1145 / 3131085.3131116$ work within specific contexts and for specific users. Motivation, for example, arises from different multiple causes [8] and differently for certain users $[7,17]$. As a result, such three components of gamification, i.e. design elements, users, and contexts, remain obscure.

Actually, some attempts to categorize game design elements for gamification have been made over the years, in order to find some correspondences between user types and design elements. Exton \& Murray [5], for example, tried to classify game design elements on the basis of theories of motivations, while Robinson \& Bellotti [15] described a taxonomy of gamification elements on the basis of different levels of expected engagement and willingness to commit time to interaction. Such attempts, however, were based exclusively on reviews of related literature. With the partial exception of [14], categorizations built on empirical data are almost absent in the current gamification debate. As a consequence, designers continue to apply a limited variety of game elements, commonly points, badges and leaderboards, indiscriminately to different domains, in the hope that they will provoke more or less the same kind of effects [13]: the lack of an empirical base, in fact, undermines any possibility of predicting the possible impact of specific design elements on user's behavior. Although some research tried to tailor gamification to specific users' characteristics [12], personalized gamified design has been limited to abstract model of behavior or motivation (e.g. player or user types), without considering the actual context in which the user is situated.

Instead, we need to know how game design elements work for situated needs, goals and motivations. This would produce more effective and satisfactory gamification designs. However, this, on the one hand, implies a thorough investigation on how specific contexts, as well as users' idiosyncrasies, might affect the effectiveness of gamification techniques: we need both qualitative and quantitative studies that might reveal the varying impacts of gamified elements, depending on the domain to which they are applied. On the other hand, it requires taking into account the continuous flux of user data which we have now at our disposal. According to a recent IBM research, 90 percent of the information available have been created in the last two years. This exponential increasing of digital data gives new life to research in the area of personalization: information about users' preferences, sentiment, beliefs, social relations, and physical context, as well as parameters describing their psychological states can now be obtained by mining data gathered 
from many heterogeneous sources. Such sources can be roughly classified in two main categories: on the one hand, the plethora of personal data collected, either manually or automatically, by personal and ubiquitous devices such as mobile phones, wearables, and sensors; on the other hand, a huge amount of textual information spread on social networks, which can be exploited to infer user interests, personality traits, emotions, and knowledge.

Grounding gamification design on empirical data might entail the possibility of creating data-driven gamified applications. In this workshop we want to explore the different meanings that a "data-driven gamification design" might have for researchers and practitioners.

First, data-driven gamification yields to design applications and services shaped on the specific context in which they are used: this might lead to create "situated" catalogs of game elements, built on empirical data, that are thought for specific domains. Second, it implies the design of gamified services tailored to the user's characteristics, also responding in real time to her changing states and needs. The possibility of exploiting data mining and machine learning to extract knowledge from raw data could also lead to an effective employment of AI techniques in gamification design. From this perspective, design could be even automated by Creative AI [3] optimizing the creation of game design elements and configurations [9]. Third, data-driven gamification means also the conduction of more rigorous evaluations to assess the impacts of gamified designs. Through online evaluations of user interaction data, and analysis on logging data of controlled experiments (A/B tests) we might get deeper insights on game design elements and users in different domains. This could turn into the design of novel, more effective and tailored gamification elements.

To summarize we are looking for:

- Gamified systems that exploit data mining, machine learning and AI techniques.

- Insights on game design elements built upon empirical data that can expand the catalog available to gamification designers and practitioners.

- Personalized gamified systems that exploit physiological, psychological, environmental, emotional and social data to provide tailored game elements to users with different characteristics.

- Domain-dependent gamified services and applications addressed to contexts like health, learning, workplace, security, crowdsourcing, and so on.

- Field evaluations of gamified systems in specific contexts of use, and new techniques to envision, design and assess gamification design techniques.

- Theoretical reflections and ethical considerations on the future of gamification enabled by the increasing availability of data.

\section{WORKSHOP TOPICS}

Topics of interest for this workshop include but are not limited to:

- Gamification approaches in a variety of domains, e.g.: education, workplace, health and e-commerce

- Empirical studies to improve gamification design
- Evaluation of gamified applications and services in specific domains

- $\mathrm{AI}$ and machine learning for gamification

- Player's data to be used in gamification design

- New theoretical approaches for gamification design

- New game design elements grounded upon empirical data

- Personalized gamification systems

- Ethical issues and critical reflections

\section{WORKSHOP OBJECTIVES}

The first objective of this workshop is to provide a shared forum for researchers interested in gamification looking for new inspirations to design novel gamified systems. We believe that researchers need a common interdisciplinary space where generating novel ideas, trying to imagine how gamification will evolve in the next years and what could we do to make it more effective and enjoyable. The long-term objective of the workshop is to support the creation of a community interested in sharing insights on gamified systems and collectively develop a new catalog of game design elements grounded upon empirical data. We hope this workshop will be a starting point for researchers to join and share their knowledge and experiences.

\section{EXPECTED OUTCOMES}

In this workshop we aim to discuss the opportunities of data-driven gamification design, also to create further insights on taxonomies and models for gamification. We want to develop strategies and recommendations on appropriate measures by developing a series of best practices. We plan for accepted manuscripts to be included in the Workshop Proceedings that will be published through CEUR Workshop Proceedings. All workshop papers must be up to six pages long in the ACM SIG format.

\section{PLANNED ACTIVITIES}

Pre-workshop preparation: The workshop website will go online before the call for participation is sent to all the major games, gamification and HCI mailing lists. The organizers will then publicize the call in their home organizations and among their peers. We will also invite directly researchers from diverse disciplines, such as UX designers, developers, data mining and data visualization experts, social scientists and psychologists, to participate. Finally, we will advertise the workshop and distribute the CFPs among the main gamification social media channels.

Conducting the workshop: The workshop will follow a full-day format. We aim to invite a keynote speaker with expertise in game design because we think gamification researcher and practitioner could benefit from a knowledge transfer. After the keynote and a coffee break, attendees will present their papers in order to introduce each other and to set a common background for further ideas and discussions. Therefore, organizers together with the participants will define three gamification design challenges as preparation for the first work group phase. In order to bridge the gap between researchers and practitioners we plan to develop concrete gamification design challenges and possible data-driven solutions in three consecutive work group phases. 
In the first group phase, participants will work individually to generate as much as possible new ideas to envision new data- or AIdriven approaches and solutions to address the design challenges individuated. After 15 minutes, they will have to collaborate for 30 minutes with their companions to develop three "best solutions", one for each design challenge to be addressed. Then, each pair will have 10 minutes to present their work and answer to all the questions (possibly critical and addressed to discover the weak points of the solution) in a presentation madness session. Each presented idea will then be voted by the remaining participants on the basis of their quality and capability of responding to the criticalness raised by the questions, gaining "points" and entering in a "leader board". At the end of the presentation/discussion session the three ideas (one for each design challenge) at the top of the leader boards will pass to the next phase.

In the second group phase of the workshop, participants will have to select one of the "winning" ideas and turn it into a concept design embedded in a narrative"critical" scenario. These scenarios could also take the form of utopias and dystopias to open spaces for reflection about the desired or undesired consequences of the idea.

In the last phase, participants will have to present their concepts and scenarios to the rest of attendees in order to generate further insights.

After the workshop: To foster the discussion even after the end of the workshop we plan to: i) include all the participants in a mailing list where they can easily discuss new ideas related to the workshop topics; ii) produce a report of the workshop to disseminate the insights emerged during the work group; iii) Submit an article to ACM Interactions with the main workshop results.

\section{SCHEDULE}

09:30 - 10:30 Keynote

10:30 - 11:00 Coffee Break

11:00 - 12:30 Introduction and paper presentations

12:30 - 14:00 Lunch

14:00 - 15:00 Group work I: idea generation and discussion

14:00 - 16:00 Group work II: data-driven solutions

16:00 - 16:30 Coffee Break

16:30 - 17:30 Group work III: presentations, discussion and conclusion

\section{INTENDED AUDIENCE}

Intended audience are researchers or practitioners in the field of $\mathrm{HCI}$, game design, AI or machine learning who applied or plan to apply gamification. We expect to gather 15-20 participants in total and 10-15 paper submissions. We will accept both position papers and research papers, case studies, future research challenges and reflections. Submissions will be selected based on their quality and capability of eliciting insights during the workshop by a PC of key researchers in the machine learning, game and gamification domains.

\section{ORGANIZERS}

Michael Meder is pursuing a $\mathrm{PhD}$ at the Distributed Artificial Intelligence Laboratory of the TU Berlin with a thesis on gamification in the workplace. His main research goal is to find solutions for the Gamification Design Problem [10,11] with machine learning on user interaction data collected in gamification field studies. He was co-chair of the three Gamification for Information Retrieval (GamifIR) workshops in 2014,2015 and 2016 at ECIR and SIGIR.

Amon Rapp is a research fellow at the university of Torino. He organized the Fictional Game Elements workshop at CHI Play 2016 and the tutorial on Games, gamification and personalization at ACM UMAP '16. He is currently a guest editor at IJHCS for a special issue on Strengthening gamification studies: Critical challenges and new opportunities. His research interests are personal informatics, behavior change and gamification design.

Till Plumbaum is a postdoctoral researcher at the Distributed Artificial Intelligence Laboratory of the TU Berlin, leading the research group Information Retrieval and Machine Learning. He is author of several conference and book publications and frequent reviewer of high ranked conferences including IUI and RecSys. He also organizes workshops covering topics such as Lifelogging and Personalization.

Frank Hopfgartner is Lecturer at University of Glasgow. His main research interest is on interactive information retrieval, recommender systems and multimedia analysis. He has co-organized workshops, chaired sessions and tutorials at various conferences. Besides, he is on the editorial board of the Information Processing \& Management Journal, serves as regular reviewer of various renowned journals and has been PC member of all leading conferences in the multimedia and information retrieval fields.

\section{REFERENCES}

[1] Gabriel Barata, Sandra Gama, Manuel J. Fonseca, and Daniel Gonçalves. 2013. Improving Student Creativity with Gamification and Virtual Worlds. In Proceedings of the First International Conference on Gameful Design, Research, and Applications (Gamification '13). ACM, New York, NY, USA, 95-98. https: //doi.org/10.1145/2583008.2583023

[2] A. Joseph Cafazzo, Mark Casselman, Nathaniel Hamming, K. Debra Katzman, and R. Mark Palmert. 2012. Design of an mHealth App for the Self-management of Adolescent Type 1 Diabetes: A Pilot Study. 7 Med Internet Res 14, 3 (08 May 2012), e70. https://doi.org/10.2196/jmir.2058

[3] Sebastian Deterding. 2016. Desperately Seeking Theory: Gamification, Design, Data, Machine Learning, and the Promise of Double Loop Learning Systems. In GamifIR 2016 workshop co-located with SIGIR 2016. 1. https://goo.gl/7pbJfs

[4] Mora et al (UOC). 2015. A literature review of gamification design frameworks - Alberto Mora, Daniel Riera, and Carina Gonzalez - Academia.edu. In 2015 7th International Conference on Games and Virtual Worlds for Serious Applications (VS-Games). http://www.academia.edu/12847611/A

[5] G Exton and L Murray. 2014. Motivation: A Proposed Taxonomy using Gamification. (2014), 1-20.

[6] Juho Hamari, Jonna Koivisto, and Harri Sarsa. 2014. Does gamification work?-a literature review of empirical studies on gamification. System Sciences (HICSS), 2014 47th Hawaii International Conference on (2014), 3025-3034. https://doi.org/ 10.1109/HICSS.2014.377

[7] Juho Hamari and J Tuunanen. 2014. Player Types: A Metasynthesis. In Transactions of the Digital Games Research Association. https:/www.hiit.fi/u/hamari/ 2013-player

[8] Yuan Jia, Bin Xu, Yamini Karanam, and Stephen Voida. 2016. Personality-targeted Gamification: A Survey Study on Personality Traits and Motivational Affordances. In Proceedings of the 34th Annual ACM Conference on Human Factors in Computing Systems - CHI '16. https://doi.org/10.1145/2858036.2858515

[9] Derek Lomas, Kishan Patel, Jodi L. Forlizzi, and Kenneth R. Koedinger. 2013. Optimizing challenge in an educational game using large-scale design experiments. Proceedings of CHI 2013 (2013), 89-98. https://doi.org/10.1145/2470654.2470668 
[10] Michael Meder and Brijnesh Johannes Jain. 2014. The Gamification Design Problem. CoRR abs/1407.0843 (2014). http://arxiv.org/abs/1407.0843

[11] Michael Meder, Till Plumbaum, and Sahin Albayrak. 2016. Learning Gamification Design - An Usability First Approach for the Enterprise Infoboard Experiment. GamifIR 2016 workshop co-located with SIGIR 2016 (2016).

[12] Rita Orji, Regan L. Mandryk, Julita Vassileva, and Kathrin M. Gerling. 2013 Tailoring Persuasive Health Games to Gamer Type. In Proceedings of the SIGCHI Conference on Human Factors in Computing Systems (CHI '13). ACM, New York, NY, USA, 2467-2476. https://doi.org/10.1145/2470654.2481341

[13] Amon Rapp. 2017. Designing interactive systems through a game lens: An ethnographic approach. Computers in Human Behavior 71 (2017), 455 - 468 https://doi.org/10.1016/j.chb.2015.02.048

[14] Amon Rapp. 2017. From Games to Gamification: A Classification of Rewards in World of Warcraft for the Design of Gamified Systems. Simulation \& Gaming 48 3 (2017), 381-401. https://doi.org/10.1177/1046878117697147

[15] David Robinson and Victoria Bellotti. 2013. A Preliminary Taxonomy of Gamification Elements for Varying Anticipated Commitment. Chi'13 (2013), 1-6. http://gamification-research.org/wp-content/uploads/2013/03/Robinson

[16] Laurentiu Catalin Stanculescu, Alessandro Bozzon, Robert-Jan Sips, and GeertJan Houben. 2016. Work and Play: An Experiment in Enterprise Gamification. In Proceedings of the 19th ACM Conference on Computer-Supported Cooperative Work \& Social Computing (CSCW '16). ACM, New York, NY, USA, 346-358. https://doi.org/10.1145/2818048.2820061

[17] Nick Yee. 2016. The Gamer Motivation Profile: What We Learned From 250,000 Gamers. In Proceedings of the 2016 Annual Symposium on Computer-Human Interaction in Play (CHI PLAY '16). ACM, New York, NY, USA, 2-2. https://doi. org/10.1145/2967934.2967937 